\title{
Efecto de dos tipos de fertilizantes y abonos en el rendimiento del repollo corazón de buey (Brassica oleracea)
}

\section{Effect of two types of fertilizers and fertilizers on the performance of the ox-heart cabbage (Brassica oleracea)}

\author{
Nestor Claumer Epiquien Torrejon ${ }^{1}$, Guillermo Idrogo Vásquez ${ }^{2}$
}

\begin{abstract}
RESUMEN
El presente estudio tuvo como objetivo determinar el efecto de la aplicación de dos tipos de fertilizantes y abonos sobre el rendimiento del repollo corazón de buey (Brassica oleracea). Para ello, se identificó el lugar donde se instaló la unidad experimental que cumplió los requisitos de homogeneidad y se empleó un diseño experimental de bloques completamente al azar (DBCA) con 9 tratamientos y 3 repeticiones. Se realizó el análisis de varianza con la prueba de Tukey $(\mathrm{p}=0,05)$. Los resultados indican que hubo diferencias significativas para peso de cabeza, longitud de planta, longitud de cabeza y diámetro de cabeza donde destaca mayormente el tratamiento T3 (Nutrifer Papa Sierra + Gran guano) con mejores promedios. Con respecto al rendimiento por parcela y hectárea se evidenció diferencias entre tratamientos donde el T3 alcanzó los mayores promedios con 17,07 kg y 105,35t/ha respectivamente, demostrando que la fertilización orgánica en interacción con la mineral influyó positivamente en el rendimiento del cultivo de repollo. Se concluye que el rendimiento por unidad experimental fue mayor con una fertilización de $25 \mathrm{~g}$ de Nutrifer Papa Sierra +7 t/ha de Gran guano, alcanzando 17,07 kg. y el rendimiento por hectárea fue superior con la aplicación de 25 gramos Molimax Papa Sierra/Planta + Guano de isla 7 t/ha, con $105,35 \mathrm{t} / \mathrm{ha}$.
\end{abstract}

Palabras clave: Fertilización, orgánico, mineral, repollo

\begin{abstract}
The research was carried out under the agroclimatic conditions of the María district, Luya province, Amazonas region, its objective was to determine the effect of the application of two types of fertilizers and fertilizers on the yield of ox heart cabbage (Brassica oleracea), for the In the execution of the investigation, the place where the experimental unit was installed that met the homogeneity requirements was identified and a completely randomized block experimental design (DBCA) with 9 treatments and 3 repetitions was used. The analysis of variance was performed with the Tukey test $(p=0,05)$. The results indicate that there were significant differences for head weight, plant length, head length and head diameter where the treatment T3 (Nutrifer Papa Sierra + Gran guano) stand out with better averages. Regarding the yield per plot and hectare, differences between treatments were evidenced where the T3 reached the highest averages with 17,07 $\mathrm{kg}$ and 105,35 $\mathrm{t} / \mathrm{ha}$ respectively, demonstrating that organic fertilization in interaction with the mineral had a positive influence on the yield of the cabbage cultivation. It is concluded that the yield per experimental unit was higher with a fertilization of $25 \mathrm{~g}$ of Nutrifer Papa Sierra $+7 \mathrm{t} /$ ha of Gran guano, reaching $17.07 \mathrm{~kg}$. and the yield per hectare was higher with the application of 25 grams Molimax Papa Sierra / Planta + Guano from the island $7 \mathrm{t} / \mathrm{ha}$, with $105.35 \mathrm{t} / \mathrm{ha}$.
\end{abstract}

Keywords: Fertilization, organic, mineral, cabbage

${ }^{1}$ Bachiller en Ingeniería Agrónoma, Universidad Nacional Toribio Rodríguez de Mendoza de Amazonas. Correo electrónico: nclaumer@gmail.com

${ }^{2}$ Docente Asociado a Tiempo Completo de la Facultad de Ingeniería y Ciencias Agrarias. Universidad Nacional Toribio Rodríguez de

Mendoza de Amazonas. Correo electrónico: guillermo.idrogo@untrm.edu.pe 


\section{INTRODUCCIÓN}

La producción de repollo en la región de Amazonas según el último reporte de producción hortofrutícola, es de $2345 \mathrm{t} /$ ha siendo superada por regiones como lima, Lambayeque, la libertad y Arequipa (Instituto Nacional de Estadística e Informática, 2014.

La importancia del repollo radica en su constante demanda durante todo el año; lo cual puede generar un requerimiento de mano de obra casi a la par de la demanda del producto. La producción de repollo bajo condiciones controladas puede estar garantizado durante todo el año; logrando el máximo rendimiento de producción de alta calidad comercial en superficies pequeñas y sobre todo aplicando tecnología relativamente baja en costos de producción (Martínez, 2016).

Nina (2014), en su investigación sobre el efecto del abonamiento con dos tipos de compost en el rendimiento de cuatro variedades de repollo (brassica oleraceal. var. capitata), mediante la aplicación de compost ME obtuvo cabezas de repollo con diámetro superiores de 21,49 cm y sin ningún tipo de enmienda logró un rendimiento de 99,42 t/ha. Portillo (2015), estudió el efecto del nitrógeno, fósforo y potasio en el cultivo de repollo, los mejores resultados fueron con las dosis de $23 \mathrm{t} / \mathrm{ha}(\sin \mathrm{N})$ y 32 $\mathrm{t} / \mathrm{ha}(\operatorname{con} \mathrm{N})$, representando un incremento del $39 \%$ en comparación del testigo, mientras que por el lado del efecto del fósforo también influyó significativamente en el rendimiento, obteniendo $21,20\left(\sin P_{2} O_{5}\right.$ y $33,70\left(\operatorname{con} P_{2} O_{5} \mathrm{t} /\right.$ ha $\left.\mathrm{t} / \mathrm{ha}\right)$ respe ctiva mente, generando un incremento del $59 \%$.

Collazos, Arista, Oyarce y Huamán2018), en su investigación sobre el efecto de la aplicación de abonos foliares y enmiendas orgánicas, en el rendimiento de repollo corazón de buey (Brassica oleracea L.), logró el mejor resultado de 14,6 y menor resultado de 12,5.

Teniendo en cuenta que la producción de repollo en nuestra región es representativa y conociendo que este cultivo en el distrito de María es producido de manera alternativa, nos propusimos evaluar el rendimiento del cultivo de repollo de la variedad corazón de Buey a base de dos fertilizantes y dos abonos considerando los fertilizantes y los abonos más utilizados en esta zona para la producción papa, esto con el fin de dejar un antecedente de la manera de cómo obtener buenos resultados en producción y tamaño que requiere el mercado del repollo.

\section{MATERIALES Y MÉTODOS}

El presente trabajo se realizó desde enero hasta junio de 2019, en el distrito de María, provincia de Luya región Amazonas, situándose entre las coordenadas geográficas -6.42889 Latitud y -77.9606 Longitud, a una altitud de $3420 \mathrm{~m}$ s.n.m. La zona presenta una temperatura media anual de $17{ }^{\circ} \mathrm{C}$, precipitación entre 1200 a $1800 \mathrm{~mm}$ anuales y humedad relativa media de 54\% (PIGARS, 2009).

Se utilizaron semillas certificadas y se almacigaron en sustrato compuesto por tierra agrícola, arena y materia orgánica en proporción $2: 1: 1$. Pasado 40 días se trasplantaron a campo definitivo, donde se probaron nueve tratamientos de fertilización (Tabla 1). El control de malezas se realizó de forma manual.

Tabla 1

Tratamientos de estudio.

\begin{tabular}{cl}
\hline Tratamientos & \multicolumn{1}{c}{$\begin{array}{c}\text { Nivel del factor } \\
\text { Representación }\end{array}$} \\
\hline $\mathrm{T} 1$ & Sin aplicación \\
$\mathrm{T} 2$ & $50 \mathrm{gr} . \mathrm{NPS}$ \\
$\mathrm{T} 3$ & $25 \mathrm{gr} . \mathrm{NPS}+110 \mathrm{gr} / \mathrm{ha} \mathrm{GG}$ \\
$\mathrm{T} 4$ & $25 \mathrm{gr} . \mathrm{NPS}+110 \mathrm{gr} / \mathrm{ha} \mathrm{GI}$ \\
$\mathrm{T} 5$ & $50 \mathrm{gr} . \mathrm{MPS}$ \\
$\mathrm{T} 6$ & $25 \mathrm{gr} . \mathrm{MPS}+110 \mathrm{gr} / \mathrm{ha} \mathrm{GG}$ \\
$\mathrm{T} 7$ & $25 \mathrm{gr} \mathrm{MPS}+110 \mathrm{gr} / \mathrm{ha} \mathrm{GI}$ \\
$\mathrm{T} 8$ & $110 \mathrm{gr} / \mathrm{ha} \mathrm{GG}$ \\
$\mathrm{T} 9$ & $110 \mathrm{gr} / \mathrm{ha} \mathrm{GI}$ \\
\hline
\end{tabular}

T1: Tratamiento 1, NPS: Nutrifer Papa Sierra, MPS: Molimax Papa Sierra, GG: Gran Guano; GI: Guano de Isla

Las variables que se evaluaron fueron altura de planta $(\mathrm{cm})$, peso de la cabeza $(\mathrm{kg})$, diámetro de cabeza $(\mathrm{cm})$ y rendimiento $(\mathrm{kg} / \mathrm{ha})$.

Se empleó un diseño de bloque completamente al azar (DBCA), con nueve tratamientos y tres repeticiones haciendo un total de 27 unidades experimentales. Los datos fueron sometidos a un análisis de varianza y prueba de Tukey $(p<0,05)$. El análisis al se realizó en el software estadístico MINITAB 15.0. 


\section{RESULTADOS}

Tabla 2

Promedio de peso de cabeza, según tratamientos de fertilización.

\begin{tabular}{cc}
\hline Tratamiento & $\begin{array}{c}\text { Peso de cabeza de } \\
\text { repollo }(\mathbf{K g})\end{array}$ \\
\hline $\mathrm{T} 1$ & $0,76 \mathrm{~d}$ \\
$\mathrm{~T} 2$ & $1,58 \mathrm{ab}$ \\
$\mathrm{T} 3$ & $1,71 \mathrm{a}$ \\
$\mathrm{T} 4$ & $1,46 \mathrm{ab}$ \\
$\mathrm{T} 5$ & $1,67 \mathrm{a}$ \\
$\mathrm{T} 6$ & $1,30 \mathrm{bc}$ \\
$\mathrm{T} 7$ & $1,60 \mathrm{ab}$ \\
$\mathrm{T} 8$ & $1,02 \mathrm{~cd}$ \\
$\mathrm{~T} 9$ & $1,42 \mathrm{ab}$ \\
\hline
\end{tabular}

Promedios que no comparten una letra son significativamente diferentes (Tukey, $\mathrm{p}<0,05$ ).

En la tabla 2 se muestra el peso de cabeza de repollo. Los resultados muestran la presencia de un primer grupo que consignaron resultados homogéneos y no presentan significación como son los seis tratamientos según orden de mérito, sin embargo, los dos primeros tratamientos T3 (Nutrifer Papa Sierra + Gran Guano) y T5 (Molimax Papa Sierra) con promedios de 1,71 y $1,67 \mathrm{~kg}$ por cabeza de repollo individualmente muestran significación estadística con los tratamientos T6 (Molimax Papa Sierra + Gran Guano), T8 (Gran Guano) y T1 (Testigo) que integran los tres siguientes grupos donde alcanzaron promedios de $1,30,1,02$ y $0,76 \mathrm{~kg}$ respectivamente.

\section{Tabla 3}

Promedio de longitud de planta, según tratamientos defertilización.

\begin{tabular}{ll}
\hline & $\begin{array}{c}\text { Tratamiento } \\
\text { Pesode cabeza de } \\
\text { repollo (Kg) }\end{array}$ \\
\hline $\mathrm{T} 1$ & $0,76 \mathrm{~d}$ \\
$\mathrm{~T} 2$ & $1,58 \mathrm{ab}$ \\
$\mathrm{T} 3$ & $1,71 \mathrm{a}$ \\
$\mathrm{T} 4$ & $1,46 \mathrm{ab}$ \\
$\mathrm{T} 5$ & $1,67 \mathrm{a}$ \\
$\mathrm{T} 6$ & $1,30 \mathrm{bc}$ \\
$\mathrm{T} 7$ & $1,60 \mathrm{ab}$ \\
$\mathrm{T} 8$ & $1,02 \mathrm{~cd}$ \\
$\mathrm{~T} 9$ & $1,42 \mathrm{ab}$ \\
\hline
\end{tabular}

Promedios que no comparten una letra son significativamente diferentes (Tukey, $\mathrm{p}<0,05$ ).
En la Tabla 3, nos muestra que existe un primer grupo con resultados homogéneos que no presentan significación donde están presentes los siete primeros tratamientos según orden de mérito, pero cabe mencionar que los tratamientos T7 (Molimax Papa sierra + Guano de Isla), T2 (Nutrifer Papa Sierra), T3 (Nutrifer Papa Sierra + Gran Guano), T5 (Molimax Papa Sierra), T9 (Guano de isla) y T4 (Nutrifer Papa sierra + Guano de Isla) con promedios de $34,83,34,70,34,57,34,37,34,27$ y $33,80 \mathrm{~cm}$ de longitud de planta individualmente muestran significación estadística con los tratamientos T8 (Gran Guano) y T1 (Testigo) que integran los dos siguientes grupos donde alcanzaron promedios de 31,77 y $28,10 \mathrm{~cm}$ respectivamente.

\section{Tabla 4}

Promedio de longitud de cabeza según tratamientos defertilización.

\begin{tabular}{cc}
\hline Tratamiento & $\begin{array}{c}\text { Longitud de cabeza } \\
\text { de repollo }(\mathbf{c m})\end{array}$ \\
\hline T1 & $16,13 \mathrm{c}$ \\
$\mathrm{T} 2$ & $22,27 \mathrm{a}$ \\
$\mathrm{T} 3$ & $22,20 \mathrm{a}$ \\
$\mathrm{T} 4$ & $21,20 \mathrm{a}$ \\
$\mathrm{T} 5$ & $22,17 \mathrm{a}$ \\
$\mathrm{T} 6$ & $20,70 \mathrm{ab}$ \\
$\mathrm{T} 7$ & $21,97 \mathrm{a}$ \\
$\mathrm{T} 8$ & $18,97 \mathrm{~b}$ \\
$\mathrm{~T} 9$ & $21,30 \mathrm{a}$ \\
\hline
\end{tabular}

Promedios que no comparten una letra son significativamente diferentes (Tukey, $\mathrm{p}<0,05$ ).

En la Tabla 4, nos muestra que existe un primer grupo con resultados homogéneos que no presentan significación donde están presentes los siete primeros tratamientos según orden de mérito, pero cabe mencionar que los tratamientos T2 (Nutrifer Papa Siera), T3 (Nutrifer Papa Sierra + Gran Guano), T5 (Moliamx), T7 (Molimax Papa sierra + Guano de Isla), T9 (Guano de Isla) y T4 (Nutrifer Papa sierra + Guano de Isla) con promedios de 22,27 22,20, 22,17, $21,97,21,30$ y $21,20 \mathrm{~cm}$ de longitud de cabeza individualmente muestran significación estadística con los tratamientos T8 (Gran Guano y T1 (Testigo) que integran los dos siguientes grupos donde alcanzaron promedios de 18,97 y $16,13 \mathrm{~cm}$ respectivamente. 
Tabla 5

Promedio de diámetro de cabeza, según tratamientos defertilización.

\begin{tabular}{cc}
\hline Tratamiento & $\begin{array}{c}\text { Diá metro de cabeza } \\
(\mathbf{c m})\end{array}$ \\
\hline T 1 & $6,70 \mathrm{c}$ \\
T2 & $9,20 \mathrm{a}$ \\
T3 & $9,37 \mathrm{a}$ \\
T4 & $8,70 \mathrm{a}$ \\
T5 & $9,27 \mathrm{a}$ \\
T6 & $8,70 \mathrm{ab}$ \\
T7 & $9,20 \mathrm{a}$ \\
T 8 & $7,83 \mathrm{~b}$ \\
T9 & $8,83 \mathrm{ab}$ \\
\hline
\end{tabular}

Promedios que no comparten una letra son significativamente diferentes (Tukey, $\mathrm{p}<0,05$ ).

En la Tabla 5, nos muestra que existe un primer grupo con resultados homogéneos que no presentan significación donde están presentes los siete primeros tratamientos según orden de mérito, pero cabe mencionar que los tratamientos T3 (Nutrifer Papa Sierra + Gran guano), T5 (Molimax Papa Sierra ), T7 (Molimax Papa sierra + Guano de Isla) y T2 (Nutrifer Papa Sierra) con promedios de 9,37, 9,27, 9,20 y 9,20 cm de diámetro de cabeza individualmente muestran significación estadística con los tratamientos T8 (Gran guano) y T1 (Testigo) que integran los dos siguientes grupos donde alcanzaron promedios de 7,83 y $6,70 \mathrm{~cm}$ respectivamente.

Tabla 6

Promedio de rendimiento por unidad experimental, según tratamientos de fertilización.

\begin{tabular}{cc}
\hline Tratamiento & Rendimient(kg) \\
\hline T 1 & $7,62 \mathrm{c}$ \\
T 2 & $15,82 \mathrm{ab}$ \\
T 3 & $17,07 \mathrm{a}$ \\
T 4 & $14,58 \mathrm{ab}$ \\
T 5 & $16,67 \mathrm{ab}$ \\
T 6 & $13,00 \mathrm{bc}$ \\
T 7 & $16,00 \mathrm{ab}$ \\
T 8 & $10,17 \mathrm{~cd}$ \\
T 9 & $14,17 \mathrm{ab}$ \\
\hline
\end{tabular}

Promedios que no comparten una letra son significativamente diferentes (Tukey, $\mathrm{p}<0,05$ ).

Tabla 6, nos indica la presencia de un primer grupo estadísticamente homogéneo conformado por los seis primeros tratamientos según orden de mérito no muestran significación, sin embargo, el tratamiento T3 (Nutrifer Papa Sierra + Gran Guano) obtuvo el mayor promedio con $17,07 \mathrm{~kg}$ mostrando significación estadística con los tratamientos T6 (Molimax Papa Sierra + Gran Guano), T8 (Gran Guano) y T1 (Testigo) que integran los tres siguientes grupos donde alcanzaron promedios de 13, 10,17 y $7,62 \mathrm{~kg}$ respectivamente.

Tabla 7

Promedio rendimiento por hectárea, según tratamientos de fertilización.

\begin{tabular}{cc}
\hline Tratamiento & Rendimiento (t/ha) \\
\hline T1 & $47,01 \mathrm{~d}$ \\
T2 & $97,63 \mathrm{ab}$ \\
T3 & $105,35 \mathrm{a}$ \\
T4 & $90,02 \mathrm{ab}$ \\
T5 & $102,88 \mathrm{ab}$ \\
T6 & $80,24 \mathrm{bc}$ \\
T7 & $98,76 \mathrm{ab}$ \\
T8 & $62,75 \mathrm{~cd}$ \\
T9 & $87,44 \mathrm{ab}$ \\
\hline
\end{tabular}

Promedios que no comparten una letra son significativamente diferentes (Tukey, $\mathrm{p}<0,05$ ).

Tabla 7, nos muestra la presencia de un primer grupo homogéneo que no presenta significación conformado por los seis primeros tratamientos según orden de mérito, sin embargo, el tratamiento T3 (Nutrifer Papa Sierra + Gran Guano) con promedio de 105,35 TM/ha muestra significación estadística con los tratamientos T6 (Molimax Papa Sierra + Gran Guano), T8 (Gran guano) y T1 (Testigo) que integran los tres siguientes grupos donde alcanzaron promedios de $80,24,62,75$ y 47,01 TM/ha respectivamente.

\section{DISCUSIÓN}

De acuerdo al peso de cabeza de repollo se obtuvo resultados que evidenciaron diferencia significativa en los tratamientos donde se aplicó Nutrifer Papa sierra + Gran guano y Molimax Papa Sierra con promedios de 1,71 y 1,67 kg por cabeza de repollo, además alcanzaron un tamaño mayor a $30 \mathrm{~cm}$. Estos resultados superan el reporte de Rengifo (2013), quien al utilizar humus y tres concentraciones de gallinaza indica que los mejores resultados se obtuvieron de la aplicación de gallinaza más humus en proporción de $1 / 5$ por metro cuadrado, con un promedio de $0,82 \mathrm{~kg}$ peso de cabeza de repollo y plantas de $20,84 \mathrm{~cm}$. Las diferencias mencionadas entre ambos reportes pueden deberse a que los mejores resultados de este estudio se obtuvieron con la aplicación de fertilizantes de síntesis química, los cuales son de más fácil y rápida asimilación por la planta, permitiendo un mejor desarrollo. Esta 
diferencia de resultados nos confirma que el abonamiento es importante para elevar la producción del repollo, por lo mismo que los resultados dado por Rengifo (2013), están por encima de $0,76 \mathrm{~kg}$ de peso de cabeza de repollo dado en nuestra investigación sin ninguna aplicación de enmienda mientras que respecto al tamaño de planta confirmamos que no siempre las plantas más grandes tienen los mejores rendimientos.

En relación del diámetro de cabeza de repollo en los resultados obtenidos existió diferencia significativa entre todos los tratamientos frente al testigo, pero el que más resaltó fue la aplicación con Nutrifer Papa Sierra + Gran Guano donde se obtuvo 9,37 cm de diámetro mientras que el testigo en donde no se aplicó ningún tipo de enmienda se obtuvo $6,70 \mathrm{~cm}$ diámetro de cabeza. Estos resultados obtenidos discrepan con Collazos et al. (2018) quienes en su investigación obtuvo $14,6 \mathrm{~cm}$ de diámetro de cabeza y el más bajo fue $12,5 \mathrm{~cm}$. Del mismo modo Nina (2014), mediante la aplicación de compost ME obtuvo cabezas de repollo con diámetro de $21,49 \mathrm{~cm}$ y sin enmienda obtuvo 19,91 cm, a pesar de la diferencia que existen en los resultados de las investigaciones, coincidimos que el abonamiento es muy significante para mejorar la producción.

Conforme a la longitud de cabeza de repollo, los resultados nos muestran que donde se agregó Nutrifer Papa Sierra en comparación con el testigo existe diferencias estadísticas donde se logró una longitud de 22,27 cm. Entonces teniendo estos resultados se discrepa con Kibar, Karaağaç, \& Kar (2015), que mediante la heterosis obtuvieron repollos con una longitud de cabeza hasta $25,30 \mathrm{~cm}$, pero en una diferente variedad. De acuerdo a esta diferencia significativa en los resultados obtenidos, se considera que a al igual que la fertilización es importante considerar la variedad o el mejoramiento genético para obtener mejores resultados en rendimiento de repollo.

En relación al rendimiento del cultivo de repollo se puede apreciar que donde se agregó Nutrifer Papa Sierra + Gran guano se obtuvo el mejor con 17,07 $\mathrm{kg} /$ parcela y $105,35 \mathrm{t} / \mathrm{ha}$ respectivamente, lo cual muestra significancia estadística frente a los tratamientos donde se aplicó Gran Guano, Guano de Isla y el testigo, entonces este uso del abonamiento orgánico nos permite coincidir con Nina (2014), quien en su investigación alcanzó unas 99,42 t/ha mediante la aplicación de compost. Del mismo modo, discrepar con Gonzales (2015), quien en su investigación sobre el efecto de cinco dosis de humus de lombriz en el cultivo de repollo var. "Corazón de buey" obtuvo los mejores resultados con 20,41 $\mathrm{kg} /$ ha- la aplicación de 10 t/ha de humus, lo cual supera a los demás tratamientos donde la cantidad de aplicación de humus fue inferior. La discrepancia es debido a que nuestros resultados están dentro de los parámetros expuesto por Benzing (2001), quien indica que los rendimientos de los abonos orgánicos en repollo llegan a un total de $40-70 \mathrm{t} / \mathrm{ha}$, mientras que lo expuesto por Gonzales (2015) está por muy debajo de estos parámetros y entonces la explicación más acertada es la diferencia de abonos de abonos y las condiciones agroclimáticas distintas.

Entonces por el lado de los resultados obtenidos por la fertilización mineral nos percatamos que son muy superiores a los obtenidos por Porras (2007), en su investigación de evaluación de dosis de fertilización nitrogenada y densidad del cultivo de repollo híbrido con $150 \mathrm{~kg} /$ ha de $\mathrm{N}$ logró $37 \mathrm{t} /$ ha de rendimiento. También Portillo (2015) sobre el cultivo repollo, obtuvo $23 \mathrm{t} / \mathrm{ha}(\sin \mathrm{N})$ y $32 \mathrm{t} / \mathrm{ha}(\operatorname{con} \mathrm{N})$, representando un incremento del $39 \%$ en comparación con el testigo, mientras que por el lado del efecto del fósforo también influyó significativamente en el rendimiento, obteniendo $21,20(\sin \quad y 33,70($ con $\quad t / h a$ respectivamente, generando $P_{2} O_{5}$ incremento $\mathrm{d} P_{2}{ }_{5}^{-}, 9 \%$. Entonces ante la discrepancia de la diferencia de nuestro mejor resultado comparado a lo expuesto por los investigadores anteriores se acepta la lógica de Thompson, (1998), quien dice que en general que el repollo responde bien al abono orgánico y facilita la disponibilidad de los nutrientes como P y K, ahí está la explicación que la aplicación de Nutrifer Papa Sierra + Gran guano sea superior de $59 \%$ al mejor resultado de abono orgánico.

\section{CONCLUSIONES}

El mayor peso de cabeza de repollo se registró con una fertilización de $25 \mathrm{~g}$ de Nutrifer Papa Sierra +7 t/ha de Gran guano, alcanzando 1,71 gramos.

La longitud de planta supero los $30 \mathrm{~cm}$ en los tratamientos que incluyeron algún plan de fertilización, superando al testigo.

La longitud de cabeza se encontró en un rango entre 18 a $22 \mathrm{~cm}$ en los tratamientos que incluyeron algún plan de fertilización, superando al testigo.

El mayor diámetro de cabeza se registró con una fertilización de $25 \mathrm{~g}$ de Nutrifer Papa Sierra +7 t/ha de Gran guano, alcanzando 9,37 cm.

El rendimiento por unidad experimental fue mayor con una fertilización de $25 \mathrm{~g}$ de Nutrifer Papa Sierra + 7 t/ha de Gran guano, alcanzando $17,07 \mathrm{~kg}$.

El rendimiento por hectárea fue superior con la aplicación de 25 gramos Molimax Papa Sierra/Planta + Guano de isla $7 \mathrm{t} / \mathrm{ha}$, con 105,35 t/ha. 


\section{REFERENCIAS BIBLIOGRÁFICAS}

Benzing, A. (2001). Agricultura orgánica: Fundamentos para la región andina. Villingen-Schwenningen, España: NeckerVerlag.

Collazos, R., Arista, J. P., Oyarce, S. K. y Huamán, E. (2018). Efecto de la aplicación de abonos foliares y enmiendas orgánicas, sobre el rendimiento de repollo corazón de buey (Brassica oleracea L.), en Chachapoyas, Amazonas. Revista de Investigación en Agroproducción Sustentable, 2(1), 28-35. http://dx.doi.org/10.25127/aps.20181.381

Gonzales, M. R. (2015). Efecto de cinco dosis de humus de lombriz en el cultivo de repollo (brassica oleracea l.) var. corazón de buey, en la zona del Alto Huallaga - Tocache [tesis de pregrado, Universidad Nacional de San Martin]. Repositorio institucional UNSM. http://repositorio.unsm.edu.pe/handle/1 1458/6 86

Kibar, B., Karaağaç, O. and Kar, h. (2015). Heterosis for yield contributing head traits in cabbage (Brassica oleracea var. capitata). Ciencia e Investigación Agraria, 42(2), 205216. http://dx.doi.org/10.4067/S0718-162020 15000200007

Nina, O. A. (2014). Efecto del abonamiento con dos tipos de preparación de compost en el rendimiento de cuatro variedades de repollo (Brassica oleracea L. var. capitata) en K'yaraCusco [tesis de pregrado, Universidad Nacional de San Antonio Abad del Cusco]. Repositorio institucional UNSAAC. http://repositorio.unsaac.edu.pe/handle/UNSA AC/979

Porras, F. J. (2007). Evaluación de dosis de fertilización nitrogenada y densidad de siembra sobre el rendimiento del cultivo de repollo (Brassica oleraceae, var capitata L.) híbrido Izalco [tesis de pregrado, Universidad Nacional Agraria]. Repositorio institucional UNA. https://repositorio.una.edu.ni/2034/

Portillo, H. J. V. (2015). Efecto de nitrógeno, fósforo $y$ potasio en el cultivo de repollo; Olopa, Chiquimula, [tesis de grado, Universidad
Rafael Landívar]. Repositorio institucional URL. http://recursosbiblio.url.edu.gt /tesisjcem/2015/06/09/Portillo-Homero.pdf

Rengifo, R. E. (2013). Utilización de humus y tres concentraciones de gallinaza y su efecto en el rendimiento de la col repollo (Brassica oleracea l.) var. Tropical Delight en bolsas de polietileno en Iquitos - Perú [tesis de pregrado, Universidad Nacional de la Amazonía Peruana]. Repositorio institucional UNAP. http://repositorio.unapiquitos.edu.pe/handle/U NAP/1885 